\section{Relation of Arachnophobia with ABO blood group system}

\author{
Muhammad Imran Qadir and Sani E Zahra* \\ Institute of Molecular Biology \& Biotechnology, Bahhauddin Zakariya University, Multan, Pakistan
}

\section{Abstract}

In 1901, ABO system of blood groups was determined by Karl Landsteiner. It is present in different organisms like rodents, primates that includes chimpanzees, bonobos etc. The blood groups type in this system depends on some genes that are specific ABO gene. Arachnophobia is the dread of creepy crawlies and different creature like spiders. Individuals suffering from arachnophobia generally feels uneasy in any place where they accept that they could harbour arachnids or their existence for example, webs. 100 samples of blood from volunteers were used for Blood group test. The Blood groups were tested and results were recorded after the test all the used kits were discarded. The results shows that there is no clear cut difference between the arachnophobic males and non-arachnophobic male's percentages, so no relation found in males. Similarly, in females both phobic and non-phobic ladies have no differences in their result values so, no relation was found. Whereas, in case of arachnophobic males and females comparison no relation was found. So, there is no relationship between $A B O$ blood group system and arachnophobia and there may be a relation between non-phobia and $A B+, B+$ blood group in males whereas, in females only $\mathrm{B}+$ blood group have relation with non-phobia.

\section{More Information}

*Address for Correspondence: Sani E Zahra, Institute of Molecular Biology \& Biotechnology, Bahhauddin Zakariya University, Multan, Pakistan, Tel: 03156521284; Email: sania.zahra963@gmail.com

Submitted: 29 July 2019

Approved: 07 August 2019

Published: 08 August 2019

How to cite this article: Qadir MJ, Zahra SE. Relation of Arachnophobia with $\mathrm{ABO}$ blood group system. J Hematol Clin Res. 2019; 3: 050-052. doi.10.29328/journal.jhcr.1001011

Copyright: (c) 2019 Qadir MI, et al. This is an open access article distributed under the Creative Commons Attribution License, which permits unrestricted use, distribution, and reproduction in any medium, provided the original work is properly cited

Keywords: Blood samples; Arachnophobia; Blood analysis

Check for updates

\section{Introduction}

The ABO blood bunch framework is utilized to indicate the existence A and B antigen either one, both, or none of them on erythrocytes. This system is very important in blood transfusion in humans. Mismatch in blood groups can result in deadly, unfavorable response after a transfusion, or an adverse or strong immune response in case of organ transplantation. The ABO blood classifications were found by Karl Landsteiner in 1901, for which he got the Nobel Prize in Physiology or Medicine in 1930 [1]. ABO blood classifications are likewise present in some different creatures, for example, rodents and primates, including chimpanzees, bonobos, and gorillas. Offspring's received blood groups from their parents. The type of blood groups in in this system is controlled by some specific gene i.e. ABO gene. This gene have 3 alleles $i, I^{A}$, and $I^{B}$. $I$ is an abbreviation of isoagglutinogen, which is another term used for antigen. The allele $I^{A}, I^{B}, i$ gives phenotypes of blood groups A, B and $O$ respectively. $I^{A}, I^{B}$ both are dominant forms so, person with ii allele have $O$ blood group. Thus people with $I^{A} I^{A}$ or $I^{A} i$ have blood group A and individuals having $I^{B} I^{B}$ or $I^{B} i$ genotype have B blood group. Whereas person having genotype $I^{A} I^{B}$ show phenotype $A B$ and individuals with genotype $i i$ have phenotype 0 [2]. Arachnophobia is the nonsensical dread or fear of creepy crawlies and different 8-legged creature like spiders. Individuals with arachnophobia will in general feel uneasy in any region they accept could harbour arachnids or that has noticeable indications of their existence, for example, webs [3]. On the off chance that arachnophobes see an insect i.e. spider, they may not enter that particular region until they have beaten the fit of anxiety that is frequently connected with their fear. A few people shout, cry, have passionate upheavals, experience inconvenience breathing, sweat, have expanded pulses, or even black out when they interact with a region close bugs or their networks. In some extraordinary cases, even an image or a practical illustration of a spider can trigger extreme dread. The dread of arachnids can be treated by any of the general strategies proposed for explicit fears. The principal line of treatment is methodical desensitization otherwise called presentation treatment or exposure therapy [4].

\section{Methodology}

\section{Project design}

100 Samples of blood from volunteers were taken in laboratory for blood group test. A question about 
arachnophobia i.e. Do they fear from spider was also asked by them individually. The project was planned and executed in Bahauddin Zakariya University (BZU) Multan at Institute of Molecular Biology and Biotechnology (IMBB).

\section{Samples design}

100 samples of blood from volunteers were taken and used for blood group testing.

\section{Methodology}

Blood testing or analysis is used to determine what sort or type of blood you have. This test is done or carried out so that one can securely donate his blood or get transfusion of blood. It is also done to check whether a substance called $\mathrm{Rh}$ factor is present on the outside of one's red blood cells or platelets. Blood type is usually based on presence or absence of certain specific proteins on the surface of (RBCs) i.e. red blood cells. These specific proteins are known as antigens. The blood group or type depends on which types passed down to individual from his parents. Blood test was done in laboratory. The test to decide ones blood group is termed as ABO typing. The samples of blood were taken and blended with antibodies against the types i.e. A \& B blood. At that point, the example is verified whether the platelets stick together. In the event that platelets stick together, it implies the blood responded with one of the antibodies. Rh typing utilizes a technique like ABO typing. At the point when blood testing was done to check whether an individual have $\mathrm{Rh}$ factor on the outside of his red platelets, the outcomes were: $\mathrm{Rh}+$ (positive), in the event that you have this cell surface protein or Rh-(negative), in the event that you don't have this cell surface protein [5]. The blood group system i.e. ABO $\&$ Rh depends on clumping or agglutination reaction. When RBCs have one or more (both) antigens and are exposed to respective antibodies, the reaction occur i.e. antigen and antibodies bind to each other and produce clumps (agglutination). In ABO system antigens are glycoproteins i.e. 0 linked in which the residues of sugar (terminal region) at the surface of the cell (RBCs) decide if the antigen is A or B. Antigen A and antibodies B are present on RBCs of individuals having $A$ blood group. In the same way antigen $B$ and antibodies A i.e. anti-A are present in Individuals with B Blood group [6]. The persons who have AB blood group have $A, B$ antigen and absence of antibodies in their serum and people with $O$ blood group have no antigen in their serum but have anti-A, anti-B antibodies in their serum HiPer $^{\circledR}$ Blood Grouping Teaching Kit is a rapid test kit and enables us to quickly identify the $\mathrm{ABO}$ blood group and also $\mathrm{Rh}$ factor that depends on presence of antigen on RBCs. Dangle the hand of volunteer down in order to increase the flow of blood in the fingers. Clean the finger before prick with spirit or alcohol (70\%). Usually middle finger was used to prick. Fingertip was pierced with sterile lancet and only a drop of blood was placed in each cavity or portion of kit. Then one drop of antiserum was dropped in each cavity. Blood drop in each cavity was blended with the help of a mixing stick. Agglutination or clumping in each portion was observed in 30 seconds. The anti-Rh takes more time in order to form clump than anti A or anti-B. Proper care is required in this procedure after noting results. The used kit and stick was discarded and results were noted [7].

\section{Results}

From table 1 after comparing the results of both arachnophobic and Non-Arachnophobic males it was found that the percentages of phobic males are lower than nonphobic males in case of all blood groups of $\mathrm{ABO}$ system except $\mathrm{O}^{-}$, in case of $\mathrm{O}^{-}$arachnophobic males have $5.27 \%$ whereas, non- phobic males have $0 \%$ so, the difference is only found in $\mathrm{O}^{-}$blood group but the difference is not as much significant. Whereas, in case of $\mathrm{B}+$ of non-phobic male the percentages of non-phobic group are relatively high i.e. $31.6 \%$ so there is relationship between non-phobic $\mathrm{B}+$ group males and nonarachnophobia. In case of $A B+$ blood group the results of non-phobic males are higher percentages than phobic group so there may be a relationship between non-phobia and $\mathrm{AB}+$ blood group.

From graph 1, after comparing the results of both arachnophobic and Non-Arachnophobic males it was found that the results of phobic males were lower than non-phobic males in case of all blood groups of $\mathrm{ABO}$ system except $\mathrm{O}^{-}$, in the case of $\mathrm{O}^{-}$blood group arachnophobic males have values $5.27 \%$ whereas, non- phobic males have $0 \%$ so, the difference is only found in $\mathrm{O}^{-}$blood group but the difference is not as much significant. So, no relationship found among males. Whereas, in case of $\mathrm{B}+$ of non-phobic male the percentages of non-phobic group are relatively high i.e. $31.6 \%$ so there is relationship between non-phobic $\mathrm{B}+$ group males and nonarachnophobia. In case of $A B+$ blood group the results of non-phobic males are higher percentages than phobic group so there may be a relationship between non-phobia and $\mathrm{AB}+$ blood group.

From table 2, it was found that the results of Arachnophobic females are lower than Non-phobic females so, there is no clear cut difference found between the percentages of both phobic and non-phobic females. So, no relationship was found in females of both phobic and non-phobic group. In case of B+ blood group the results of non-phobic females are higher than the phobic group so there may be a relationship between the non-phobia and $\mathrm{B}+$ blood group.

\begin{tabular}{|c|c|c|}
\hline \multicolumn{2}{|c|}{ Table 1: Relation of Arachnophobia with (ABO) } & Blood groups system in Males. \\
\hline Blood Group & Arachanophobic Males & Non-Arachanophobic Males \\
\hline$A+$ & $10.5 \%$ & $10.5 \%$ \\
\hline$A^{-}$ & $0 \%$ & $0 \%$ \\
\hline$B+$ & $5.27 \%$ & $31.6 \%$ \\
\hline$B^{-}$ & $0 \%$ & $0 \%$ \\
\hline$O^{-}$ & $10.5 \%$ & $10.5 \%$ \\
\hline$O^{-}$ & $5.27 \%$ & $0 \%$ \\
\hline$A B+$ & $0 \%$ & $15.8 \%$ \\
\hline$A B^{-}$ & $0 \%$ & $0 \%$ \\
\hline Total & $31.54 \%$ & $68.4 \%$ \\
\hline
\end{tabular}


From graph 2, it was found that the results of phobic females are lower than Non-phobic females so, there is no clear cut difference between the percentages of both phobic and non-phobic females. So, no relationship was found in females of both phobic and non-phobic group. In case of B+ blood group the results of non-phobic females are higher than the phobic group so there may be a relationship between the non-phobia and B+ blood group.

From table 3, after comparing results of phobic group individuals, we found that the Females of arachnophobia having B+ blood group have $16.0 \%$ results whereas in case of males $5.27 \%$ results found so, in female's relation found in B+ blood group but no significant differences found and in $\mathrm{O}^{-}$blood group individuals males i.e. phobic have results values $5.27 \%$ and in females values found were $0 \%$ but the difference in results was not as much significant so in males no relation was found.

From graph 3, after comparing results of phobic group individuals, we found that the Females of arachnophobia having B+ blood group have $16.0 \%$ results whereas in case of males $5.27 \%$ results found so, in female's relation found

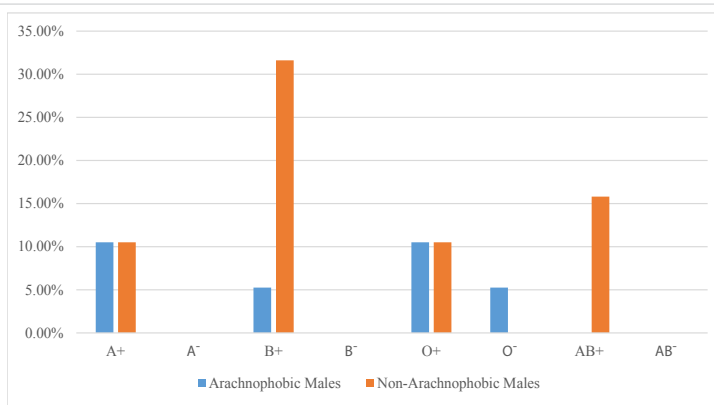

Graph 1: Relation of Arachnophobia with (ABO) Blood groups system in Males.

Table 2: Relation of Arachnophobia with (ABO) Blood groups system in Females. Blood Group Arachanophobic Females Non-Arachanophobic Females

\begin{tabular}{|c|c|c|}
\hline $\mathrm{A}+$ & $8.6 \%$ & $8.7 \%$ \\
\hline $\mathrm{A}^{-}$ & $1.2 \%$ & $0 \%$ \\
\hline $\mathrm{B}+$ & $16.0 \%$ & $28.5 \%$ \\
\hline $\mathrm{B}^{-}$ & $1.2 \%$ & $1.2 \%$ \\
\hline $\mathrm{O}+$ & $9.8 \%$ & $16.2 \%$ \\
\hline $\mathrm{O}^{-}$ & $0 \%$ & $1.2 \%$ \\
\hline $\mathrm{AB}+$ & $2.5 \%$ & $4.9 \%$ \\
\hline $\mathrm{AB}^{-}$ & $0 \%$ & $0 \%$ \\
\hline Total & $39.3 \%$ & $60.7 \%$ \\
\hline
\end{tabular}

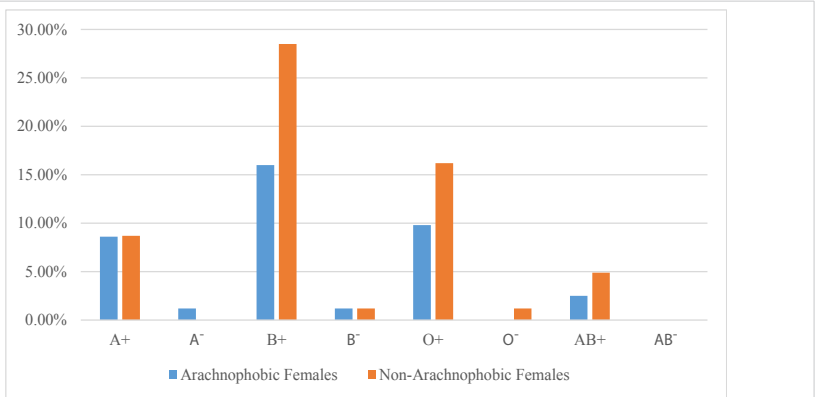

Graph 2: Relation of Arachnophobia with (ABO) Blood groups system in Females.
Table 3: Relation of Arachnophobia with (ABO) Blood Groups System in Arachnophobic Males and Females.

\begin{tabular}{|c|c|c|}
\hline Blood Group & Arachanophobic Males & Arachanophobic Females \\
\hline $\mathrm{A}^{+}$ & $10.5 \%$ & $8.6 \%$ \\
\hline $\mathrm{A}^{-}$ & $0 \%$ & $1.2 \%$ \\
\hline $\mathrm{B}+$ & $5.27 \%$ & $16.0 \%$ \\
\hline $\mathrm{B}^{-}$ & $0 \%$ & $1.2 \%$ \\
\hline $\mathrm{O}+$ & $10.5 \%$ & $9.8 \%$ \\
\hline $\mathrm{O}^{-}$ & $5.27 \%$ & $0 \%$ \\
\hline $\mathrm{AB}^{-}$ & $0 \%$ & $2.5 \%$ \\
\hline $\mathrm{AB}^{-}$ & $0 \%$ & $0 \%$ \\
\hline
\end{tabular}

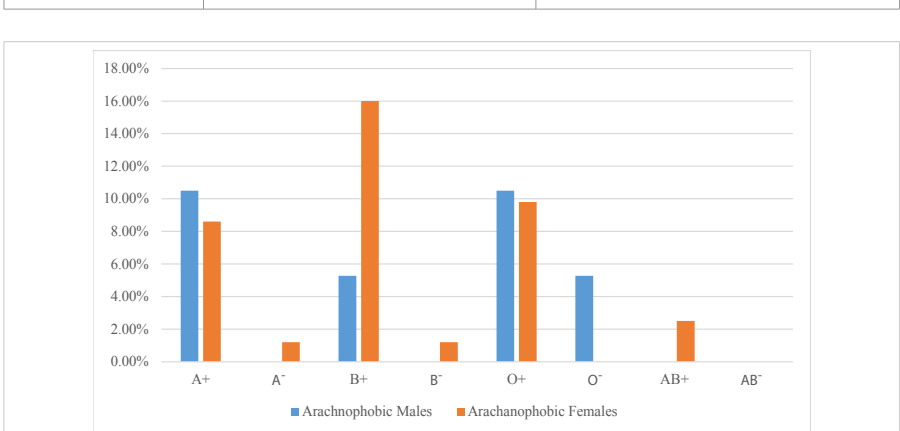

Graph 3: Relation of Arachnophobia with (ABO) Blood Groups System in Arachnophobic Males and Females.

in $\mathrm{B}+$ blood group but the difference in percentages is not as much so no significant difference found and in males no relation was found.

\section{Conclusion}

There is no relationship between Arachnophobia and ABO system of blood groups and there may be a relation between non-phobia and $\mathrm{AB}+\mathrm{B}+$ blood group in males whereas, in females only B+ blood group have relation with non-phobia.

\section{References}

1. Watkins $W$. The ABO blood group system: historical background. Transfusion medicine. 2001; 11: 243-265. PubMed: https://www. ncbi.nlm.nih.gov/pubmed/11532183

2. Hassawi DS. Allele frequency and molecular genotypes of $A B O$ blood group system in a Jordanian population. J Med Sci. 2007; 7: 51-58.

3. Granado LC, Ranvaud RP Jr. A spiderless arachnophobia therapy: comparison between placebo and treatment groups and six-month follow-up study. Neural plasticity. 2007. PubMed: https://www.ncbi. nlm.nih.gov/pubmed/17713595

4. Bouchard S, Côté S, St-Jacques J, Robillard G, Renaud P. Effectiveness of virtual reality exposure in the treatment of arachnophobia using 3D games. Technology and health care. 2006; 14: 19-27. PubMed: https://www.ncbi.nlm.nih.gov/pubmed/16556961

5. Ogasawara K, Yabe R, Uchikawa M, Saitou N, Bannai $M$, et al. Molecular genetic analysis of variant phenotypes of the $A B O$ blood group system. Blood. 1996; 88: 2732-2737. PubMed: https://www. ncbi.nlm.nih.gov/pubmed/8839869

6. Al-Arrayed S, Shome DK, Hafadh N, Amin S, Al Mukhareq H, et al. ABO blood group and Rhd phenotypes in Bahrain: Results of screening school children and blood donors. Bahrain Med Bull. 2001; 23: 112-115.

7. Levine DM, Green LW, Deeds SG, Chwalow J, Russell RP, et al. Health education for hypertensive patients. JAMA. 1979; 241: 1700-1703. PubMed: https://www.ncbi.nlm.nih.gov/pubmed/430732 